\title{
Targeted therapy and immunotherapy for platinum-refractory advanced ovarian adenosquamous carcinoma: a case report
}

This article was published in the following Dove Press journal:

OncoTargets and Therapy

Anji Li, ${ }^{1,2, *}$ Shuai Sun, ${ }^{2, *}$ Tao Song, ${ }^{3} \mathrm{Xi} \mathrm{Li,}{ }^{2}$ Wen Cheng, ${ }^{2}$ Ruipin Yao, ${ }^{2}$ Danying Zhang, ${ }^{2}$ Zailong Cai, ${ }^{4}$ Jie Zhang, ${ }^{5}$ Dongxia Zhai, ${ }^{2}$ Chaogin $\mathrm{Yu}^{2}$

'Shanghai University of Traditional Chinese Medicine, Shanghai 20 I203, People's Republic of China; ${ }^{2}$ Department of Gynecology of Traditional Chinese Medicine, Changhai Hospital, Second Military Medical University, Shanghai 200433, People's Republic of China; ${ }^{3}$ Department of Radiology, Changhai Hospital, Second Military Medical University, Shanghai 200433, People's Republic of China; ${ }^{4}$ Department of Biochemistry and Molecular Biology, Second Military Medical University, Shanghai 200433, People's Republic of China; ${ }^{5}$ Department of Assisted Reproduction, Shanghai Ninth People's Hospital, Shanghai Jiao Tong University School of Medicine, Shanghai 2000II, People's Republic of China

*These authors contributed equally to this work

Correspondence: Chaoqin Yu; Dongxia Zhai

Department of Gynecology of Traditional Chinese Medicine, Changhai Hospital, Affiliated to Second Military Medical University, 168 Changhai Road, Shanghai 200433, People's Republic of China

Tel +86 2I I38 I839 0984;

+862113761189520

Email chqyu8I@I63.com;

zhaidongx@163.com
Background: Ovarian adenosquamous carcinoma is an extremely rare type of ovarian histology. Platinum-refractory disease is also uncommon, but can be fatal because of the lack of available treatment options. To date, there is no study or case report on platinum-refractory ovarian adenosquamous carcinoma or its relevant treatment.

Case presentation: Herein, we report the case of a 38-year-old Chinese woman with platinumrefractory advanced ovarian adenosquamous carcinoma who received clinical benefit from poly adenosine diphosphate ([ADP] ribose) polymerase and programmed death-1 inhibitors after failure of prior multiline chemotherapies and antiangiogenic agents. The targeted therapy and immunotherapy-controlled disease deterioration and improved performance status. Thus far, the patient has survived longer than 15 months, and she is taking nivolumab as maintenance treatment.

Conclusion: Targeted therapy and immunotherapy may be options for rare categories of ovarian cancer, but this warrants more clinical evidence of efficacy and toxicity.

Keywords: ovarian adenosquamous carcinoma, platinum-refractory, olaparib, nivolumab, pembrolizumab

\section{Background}

Ovarian cancer is the leading cause of death among gynecologic malignancies. Adenosquamous carcinoma is an extremely rare primary malignant tumor of the ovary, accounting for $<1 \%$ of all ovarian malignancies. ${ }^{1}$ We searched the PubMed database (up to April 14, 2018) using the search terms "ovarian adenosquamous carcinoma" and "'adenosquamous carcinoma' and 'ovary." Our search generated 11 articles, most of which were case reports without an established therapeutic regimen for ovarian adenosquamous carcinoma.

Standard initial multiple modality treatment for ovarian cancer includes primary debulking surgery followed by platinum-based chemotherapy regimens. ${ }^{2}$ However, about $20 \%-25 \%$ of patients with ovarian cancer relapse during first-line chemotherapy; ${ }^{3}$ these cases are classified as platinum-refractory by the Gynecologic Oncology Group. Platinum-refractory ovarian cancer is usually fatal because there are no other treatment options. No standard second-line or beyond chemotherapy regimens are superior in safety or efficacy ${ }^{4}$ for platinum-refractory ovarian cancer.

To date, there is no study or case report on platinum-refractory ovarian adenosquamous carcinoma or any relevant treatment options. However, there may be targeted treatment options that have shown efficacy in other tumor types. Tumor angiogenesis plays a pivotal role in the growth and metastasis of ovarian cancer, 
mediated by vascular endothelial growth factor (VEGF) binding and signaling through cell surface VEGF receptors. Approximately $10 \%-15 \%$ of patients with ovarian cancer have deleterious germline BRCAmutations. ${ }^{5}$ Programmed death ligand 1 (PD-L1), an immune checkpoint receptor ligand, is expressed in many types of human tumors and allows the tumor to escape the host immune system via programmed cell death-1 (PD-1) signaling. These signaling pathways may represent new treatment options for ovarian cancer. We present a woman with platinum-refractory advanced ovarian adenosquamous carcinoma treated using targeted and immune agents after failure to respond to multiple chemotherapies.

\section{Case presentation}

A 38-year-old woman was admitted to the Changhai Hospital in Shanghai on August 22, 2016, for abdominal aching pain and lumbago. She had been previously diagnosed with ovarian endometrial cysts through ultrasound at the Obstetrics and Gynecology Hospital of Fudan University in 2009. We determined that the patient had a mixed mass $7.0 \times 5.2 \times 6.5 \mathrm{~cm}^{3}$ in the left adnexal area and moderate ascites. The patient underwent debulking surgery on September 27, 2016, including curettage, total hysterectomy, bilateral adnexectomy, greater omentum resection, and appendectomy. Pathological examination revealed endometrioid adenocarcinoma with moderate differentiation; however, intermixed squamous differentiation in partial region was observed in the result of consultation from Fudan University Shanghai Cancer Center. Referring to immunohistochemical reevaluation, which was CAM5.2 (+), CK7 (+), PAX8 $(+)$, CK20 (+) partly, Ki-67 (50\%), vimentin (+) partly, P53 $(-)$, WT1 (-), CD10 (+) partly, P63 (+) partly, $\beta$-catenin (+), ER (-), PR (-), and P16 (+) partly, adenosquamous carcinoma was confirmed ultimately (Figure 1). The patient's serum
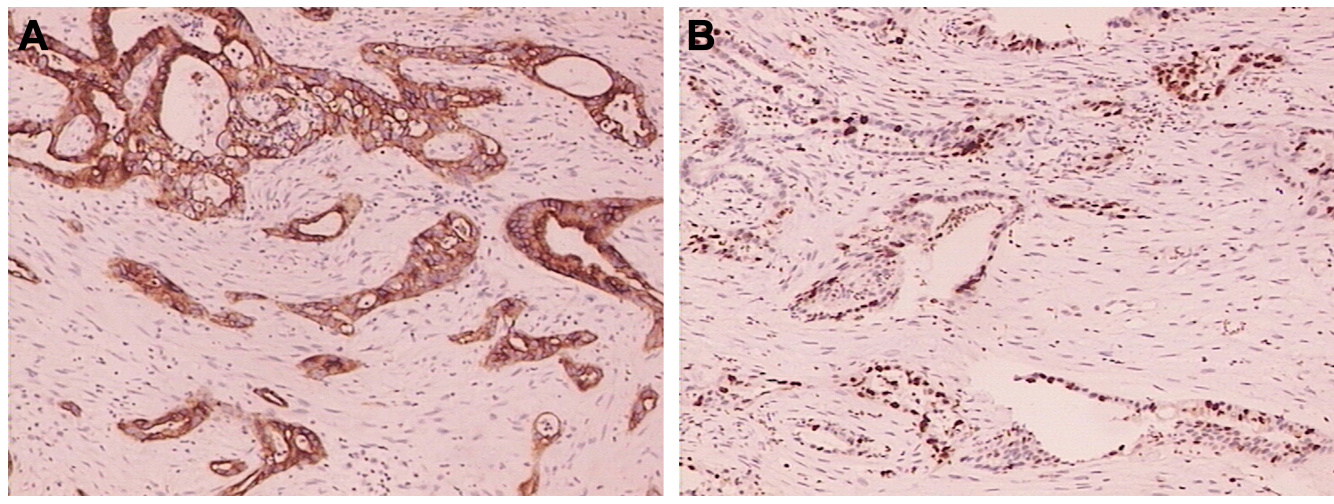

CA125 level was $245.00 \mathrm{U} / \mathrm{mL}$ and her serum CA19-9 level exceeded 1,200 U/mL on August 22, 2016, preoperatively, and they had decreased to 8.9 and $20.8 \mathrm{U} / \mathrm{mL}$, respectively, on October 19, 2016, after surgery. She was diagnosed with International Federation of Gynecology and Obstetrics 2010 stage IIA disease according to the pathology and treated with intravenous docetaxel $\left(75 \mathrm{mg} / \mathrm{m}^{2}\right)$ and intravenous united intraperitoneal carboplatin (area under the cute $=5$, on days 1 and 2) for 2 cycles. During the first cycle, the serum CA19-9 increased over the normal range (Figure 2). The response evaluation before the third cycle indicated PD according to the Response Evaluation Criteria in Solid Tumors 1.0 criteria. The patient's positron emission tomography-computed tomography results revealed metastases in the apex of the left lung, retroperitoneal lymph nodes, left armpit, and right side of the pelvic cavity, which was further clarified by pelvic magnetic resonance imaging (MRI; Figure 3A). In addition, her serum CA19-9 level almost tripled within a month.

After her first relapse, the patient was treated with intravenous paclitaxel $\left(135 \mathrm{mg} / \mathrm{m}^{2}\right)$ and nedaplatin $\left(80 \mathrm{mg} / \mathrm{m}^{2}\right)$ as second-line chemotherapy. During the treatment, the patient's serum CA19-9 level decreased to within the normal range. However, as with the first-line chemotherapy, after 2 cycles, her serum CA19-9 level was found to be increased to $43.41 \mathrm{U} / \mathrm{mL}$, and pelvic MRI indicated that the mass in the right side of the pelvic cavity had increased in size since the previous report (Figure 3B). We recommended that the patient be administered the antiangiogenic therapy bevacizumab. She received 1 cycle of epirubicin $\left(48 \mathrm{mg} / \mathrm{m}^{2}\right)$, cyclophosphamide ( $4 \mathrm{mg} / \mathrm{kg})$, and bevacizumab $(15 \mathrm{mg} / \mathrm{kg}$ ), which was discontinued because multiple new metastases were observed by liver MRI just a month later (Figure 4A). The patient then switched antiangiogenic therapies from bevacizumab to oral apatinib (500 mg/d) on April 6, 2017. However, apatinib treatment did not adequately control the

Figure I Immunohistochemical analysis showing (A) CAM5.2 (+) and (B) Ki-67 (50\%). 


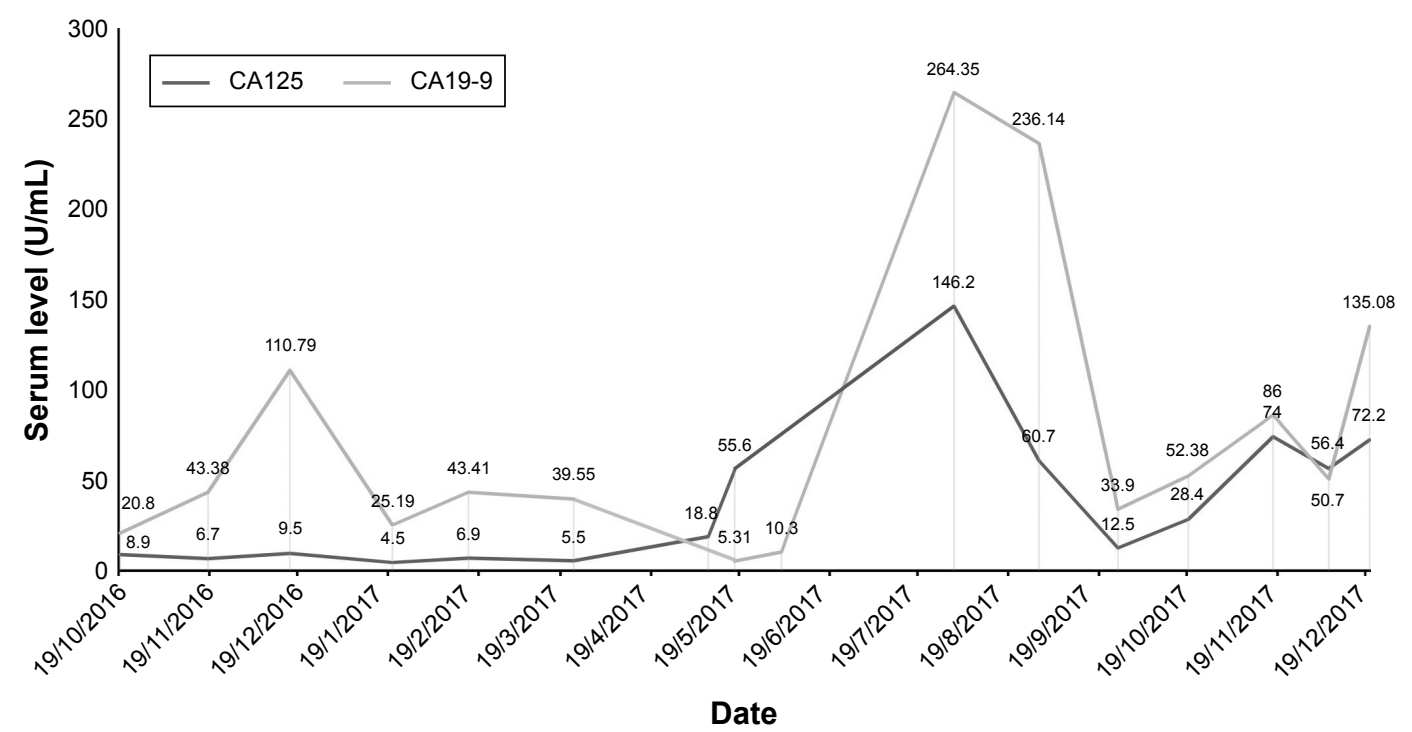

Figure 2 Serum CAI9-9 and CAI 25 levels during treatment.

disease, and the patient experienced grade 2 fatigue, handfoot skin reaction, and proteinuria, which were not well managed. Her Eastern Cooperative Oncology Group performance status was 3 . The patient stopped apatinib treatment after a month because of the side effects.

The patient underwent whole-exome sequencing by gene capture, high-throughput sequencing, and real time quantitative polymerase chain reaction (RT-Q-PCR) in April 2017, which revealed moderate plasma expression of $P D-L 1$ (Figure 5) and mutations in BRCA1 and BRCA2 at 7\% and 5\% frequency, respectively. This indicated that the patient might be sensitive to PD-1/PD-L1 and poly (ADP-ribose) polymerase (PARP) inhibitors. As the relevant medicines have not been approved on the Chinese mainland, the patient went to Hong Kong Integrated Oncology Centre to seek further therapy.

In this medical institution, the patient was treated with olaparib (300 mg, bid) and pembrolizumab (100 mg, q21d) beginning May 9, 2017, and the dosage of olaparib was increased (350 mg, bid) a month and a half later. After 4 cycles, pelvic MRI showed that the mass in the right side of the pelvic cavity had been reduced approximately $65 \%$ (Figure 3C).

In the first cycle of this regimen, her serum CA19-9 level decreased to $5.31 \mathrm{U} / \mathrm{mL}$. However, during following cycles, the patient's serum CA19-9 and CA125 levels gradually increased to all-time highs of 264.35 and $146.2 \mathrm{U} / \mathrm{mL}$, respectively. Liver MRI indicated an increased number of metastases and enlarged lymph nodes in the retroperitoneum and both the lesser and greater curvature of the stomach on August 2, 2017 (Figure 4B). The patient was then switched to nivolumab (160 mg, q14d) and pazopanib (600 mg, qd) for 8 cycles beginning August 7, 2017. The patient's serum CA19-9 and CA125 level decreased sharply and returned to within the normal range in 2 months, followed by a slow
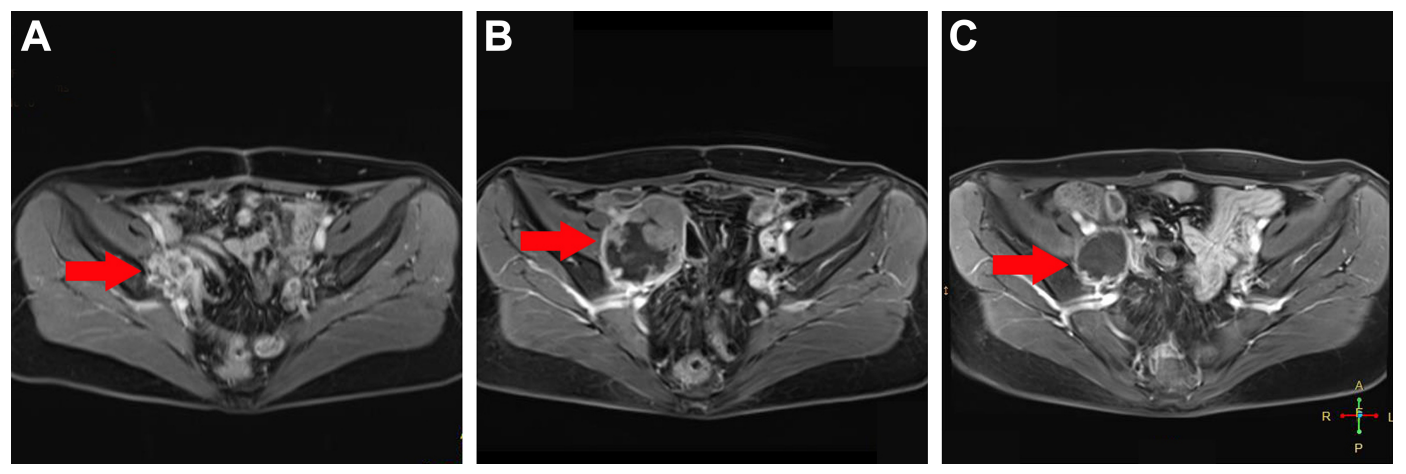

Figure 3 Pelvic MRI showing the right side pelvic cavity metastasis.

Notes: (A) After first-line chemotherapy (December 20, 2016). The new metastasis was $2.1 \times 2.8 \mathrm{~cm}^{2}$ in size. (B) Prior to olaparib and pembrolizumab treatment (March 25 , 2017). The metastasis was $5.3 \times 7.0 \mathrm{~cm}^{2}$ in size. (C) After olaparib and pembrolizumab treatment (August I, 20I7). The metastasis was $3.3 \times 4.0 \mathrm{~cm}^{2}$ in size.

Abbreviation: MRI, magnetic resonance imaging. 

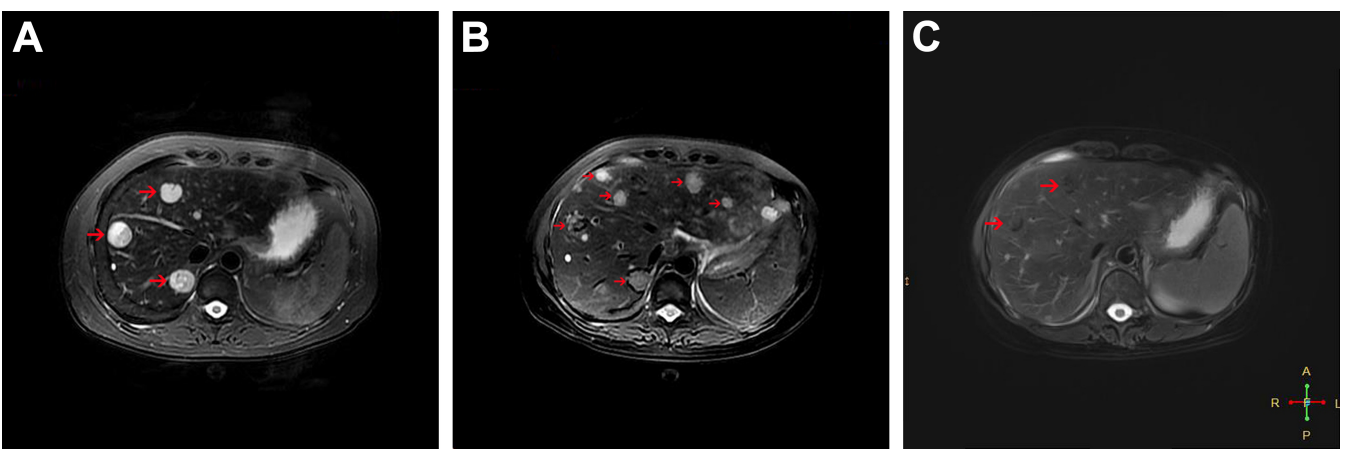

Figure 4 Liver MRI showing multiple metastases.

Notes: (A) After I cycle of epirubicin, cyclophosphamide, and bevacizumab treatment (March 27, 20I7). The metastases were first detected, and the largest was $2.5 \mathrm{~cm}$ in diameter. (B) After 4 cycles of olaparib and pembrolizumab treatment (August 2, 2017). The number of metastases increased tremendously, and the largest was $2.5 \mathrm{~cm}$ in diameter. (C) After 6 cycles of nivolumab and pazopanib treatment (October 22, 2017). The metastases diminished in volume and the largest was $2.3 \mathrm{~cm}$ in diameter. Abbreviation: MRI, magnetic resonance imaging.

increase (Figure 2). After 6 cycles, liver MRI revealed that the metastases in the retroperitoneal lymph nodes and both the lesser and greater curvature of the stomach had shrunk (Figure 4C). During the treatment, the patient felt pain in the neck, and cervical MRI showed new metastases in the C4-C7 vertebral body and part of the adnexa. The 2 agents were administered until her serum CA19-9 and CA125 level increased remarkably again on November 17, 2017. On November 21, 2017, the doctors at the Hong Kong Integrated Oncology Centre administered a combination of nivolumab with nab-paclitaxel (270 mg, q14d). The patient has received 3 cycles of this regimen and has seen a decline in the serum level of the tumor markers. Since receiving immunotherapies, the patient has experienced pneumonia and intermittent moderate fever, which were controlled by antibiotics, and her Eastern Cooperative Oncology Group (ECOG) performance status was 1. Thus far, she has survived for more than 15 months. The patient provided written informed consent for the publication of the case details and the accompanying images.

\section{Discussion and conclusion}

Approximately $80 \%$ of ovarian cancer patients go into remission after standard initial multiple modality treatment,

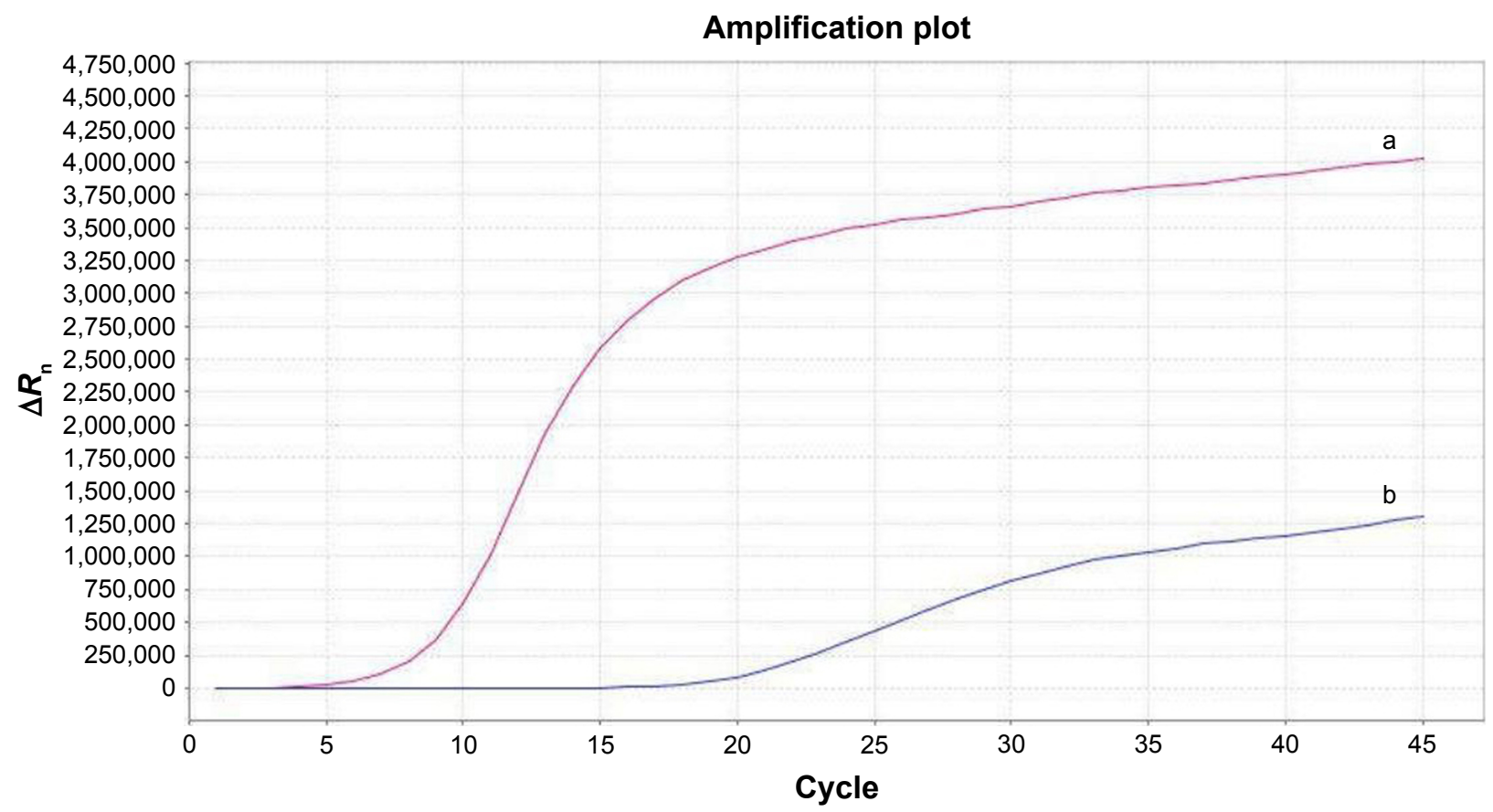

Figure 5 Moderate expression of PD-LI detected by RT-Q-PCR.

Notes: Curve $a$ is the sample and curve $b$ is the reference. $C_{t} a=17.22, C_{t} b=4.77, \Delta C_{t}=12.45$.

Abbreviation: PD-LI, programmed death ligand I; RT-Q-PCR, real time quantitative polymerase chain reaction. 
including primary debulking surgery followed by platinum chemotherapy. ${ }^{2,6}$ Platinum sensitivity is a major determinant of prognosis and, therefore, patients with platinum-refractory disease have poor prognosis. Platinum-refractory ovarian cancer patients have shorter progression-free survival and overall survival (OS) than progressive platinum-resistant patients with ovarian cancer.?

Ovarian endometriosis can lead to squamous differentiation in endometrioid adenocarcinomas, ${ }^{8}$ which are classified as adenosquamous carcinomas if they are malignant. Despite only part of the tumor consisting of malignant squamous cells, adenosquamous carcinoma was confirmed by immunohistochemical reevaluation in this case. Previous reports about ovarian adenosquamous carcinomas, almost all case reports, have been unable to determine prognosis of this type of malignant tumor. In the 5 cases where outcome was assessed, reported by Shoji et al, ${ }^{9}$ the median OS was 12 months (range: 3-36 months), and 4 of the 5 patients with stage Ic disease showed recurrence and died within 13 months. Despite adverse prognostic factors, which were platinum-refractory and advanced-stage disease (stage IIa), the patient's OS has exceeded 15 months in our case.

Tumor angiogenesis, mediated through VEGF and VEGF receptors, plays a pivotal role in the growth and metastasis of ovarian cancer. ${ }^{10}$ Bevacizumab, a monoclonal anti-VEGF-A antibody targeting tumor angiogenesis, has been investigated and widely adopted for cancer treatment in both first-line and recurrent settings. ${ }^{11}$ Apatinib is one of the latest oral small molecule tyrosine kinase inhibitors that inhibits VEGFR-2 and has encouraging preclinical and clinical data in the treatment of ovarian cancer. ${ }^{12,13}$ Pazopanib is also an oral small molecule tyrosine kinase inhibitor that inhibits VEGFR-1, -2, and -3. In a Phase III randomized clinical trial, pazopanib maintenance therapy provided a median improvement of 5.6 months (hazard ratio, 0.77) in progression-free survival in patients with advanced ovarian cancer who had not progressed after first-line chemotherapy. ${ }^{14}$ We used bevacizumab and apatinib successively combined with chemotherapy, but there seemed to be little response in this patient. The exact effect of pazopanib in combination with nivolumab cannot be determined, although taking into account previous experience, we would be inclined to ascribe a greater effect to nivolumab. El Helali et $\mathrm{al}^{15}$ demonstrated that acquired platinum resistance could be associated with an improved response to antiangiogenic agents; however, as far as we know, anti-angiogenesis-targeted platinum-refractory ovarian cancer remains to be investigated.
Olaparib is a PARP inhibitor approved by the US Food and Drug Administration (FDA) as monotherapy for ovarian cancer patients with germline $B R C A$ mutations who have been treated with 3 or more prior lines of chemotherapy. This approval was primarily based on a clinical trial involving 193 patients with platinum-resistant epithelial ovarian cancer (relapse within 6 months of platinum therapy) rather than patients with platinum-refractory disease. ${ }^{16}$ The efficacy of olaparib is associated with platinum sensitivity. Another indication from the European Medical Agency described platinum sensitivity as a condition and allowed olaparib monotherapy in patients with relapsed BRCA1/2-mutant epithelial ovarian, fallopian tube, or primary peritoneal cancers. In a cohort of 50 ovarian cancer patients with germline $B R C A$ mutations who received olaparib, the clinical benefit rate was only $23 \%$ in patients with platinum-refractory disease, compared with $69 \%$ in platinum-sensitive patients. ${ }^{17}$

PD-1, an immune checkpoint receptor expressed on $\mathrm{T}$ lymphocytes, is overexpressed in many types of human tumors in order to aid escape from the host immune system via PD-1/ PD-L1 signaling. Nivolumab and pembrolizumab, anti-PD-1 monoclonal antibodies, block PD-1 signaling, and have been used to treat many tumors. Both nivolumab and pembrolizumab have been approved by the FDA for the treatment of metastatic melanoma. ${ }^{18,19}$ The FDA has also approved pembrolizumab as first-line therapy and beyond for patients with metastatic non-small-cell lung cancer whose tumors express PD-L1. ${ }^{20}$ To the best of our knowledge, only a few Phase II trials of pembrolizumab in recurrent ovarian cancer are ongoing or completed, some of which focus on platinum-resistant cases. ${ }^{21-23}$ In a Phase II trial involving 20 nivolumab-treated patients with platinum-resistant ovarian cancer, the best overall response was $15 \%$, and the disease control rate was $45 \% .{ }^{24}$ Sun et $\mathrm{a}^{25}$ found that nivolumab increases the antitumor effects of cisplatin in platinum-resistant ovarian cancer cells by inducing apoptosis and cell-cycle arrest in cancer cells. A large amount of data about similar indications, efficacy, and toxicity of nivolumab and pembrolizumab indicate their interchangeability. ${ }^{26}$ Because anti-PD-1 mAbs nonspecifically activate T-cells, immune-mediated damage of tissue or immune-related adverse events can occur. ${ }^{27}$ Hypophysitis, colitis, hepatitis, pneumonitis, and rash are the most common adverse effects of combining immunotherapy with other therapies, but these are usually reversible. A meta-analysis demonstrated that patients treated with PD-1 inhibitors showed a significantly lower rate of grade 3-4 adverse effects than did patients receiving chemotherapy. ${ }^{28}$ In our case, the patient's performance status improved compared to her performance status during other therapies, and she did not experience toxicities generally observed during 
treatment with conventional antitumor chemotherapy. She did contract pneumonia, which was well managed.

Strickland et $\mathrm{al}^{29}$ demonstrated that BRCA1/2-mutant ovarian cancers exhibit increased $\mathrm{CD}^{+}$and $\mathrm{CD} 8^{+}$TILs and increased PD-1/PD-L1 expression in the tumor microenvironment. Cross talk between PARP inhibitors and tumorassociated immunosuppression supports the combination of PARP inhibitors and PD-L1/PD-1 immune checkpoint blockade as a potential therapeutic approach. ${ }^{30}$ The patient received benefit from the combined regimen after little response to platinum chemotherapy, non-platinum chemotherapy, and angiogenesis inhibitors, and she was facing rapid disease progression. However, this patient seemed to be progressively resistant to these drugs. Multiple resistance mechanisms against PARP inhibitors have been described, including somatic mutations in $53 \mathrm{BP} 1$, upregulation of drug efflux transporters such as $\mathrm{PgP}$, and somatic mutations in $B R C A$ genes leading to restoration of the open reading frame and thus BRCA function. ${ }^{31}$ As far as we know, there is no report of resistance to PD-1/PD-L1 inhibitors.

There is no established chemotherapy regimen for ovarian adenosquamous carcinoma and no standard second-line and beyond chemotherapy regimen for platinum-refractory ovarian cancer. In our case, targeted therapies and immunotherapies were far superior to routine chemotherapy in controlling deterioration and improving performance status, leading to a prolongation of OS. We expect that more evidence of the efficacy and safety of single or combined regimens including targeted therapies and immunotherapies will be accumulated for rare categories of ovarian cancer in the future.

\section{Data sharing statement}

The data used in the case are available from the corresponding author on reasonable request.

\section{Acknowledgments}

This work was supported by the National Natural Science Foundation of China (No 81603651). The authors thank the patient and her husband for their participation in this study and for the patients' agreement to the publication of the report. We would like to thank Editage [www.editage.cn] for English language editing.

\section{Author contributions}

AJL and SS drafted the manuscript. TS, XL, WC, RPY, and DYZ participated in collation of the clinical data. ZLC, JZ, DXZ, and CQY critically revised the paper. All authors contributed toward data analysis, drafting and revising the paper and agree to be accountable for all aspects of the work.

\section{Disclosure}

The authors report no conflicts of interests in this work.

\section{References}

1. Lee JY, Noo SM, Cho NH, et al. A rare case of primary adenosquamous carcinoma arising from ovary. J Women's Med. 2010;3:126-129.

2. Morgan RJ Jr, Armstrong DK, Alvarez RD, et al. Ovarian cancer, version 1.2016: clinical practice guidelines in oncology. J Natl Compr Canc Netw. 2016;14(9):1134-1163.

3. Bookman MA. Standard treatment in advanced ovarian cancer in 2005 : the state of the art. Int J Gynecol Cancer. 2005;15(Suppl 3):212-220.

4. Oronsky B, Ray CM, Spira AI, Trepel JB, Carter CA, Cottrill HM. A brief review of the management of platinum-resistant-platinumrefractory ovarian cancer. Med Oncol. 2017;34(6):103-109.

5. Zhang S, Royer R, Li S, et al. Frequencies of BRCA1 and BRCA2 mutations among 1,342 unselected patients with invasive ovarian cancer. Gynecol Oncol. 2011;121(2):353-357.

6. Jayson GC, Kohn EC, Kitchener HC, Ledermann JA. Ovarian cancer. Lancet. 2014;384(9951):1376-1388.

7. Sayal K, Gounaris I, Basu B, et al. Epirubicin, cisplatin, and capecitabine for primary platinum-resistant or platinum-refractory epithelial ovarian cancer: results of a retrospective, single-institution study. Int J Gynecol Cancer. 2015;25(6):977-984.

8. Yu CQ, Yu J, Han J, Zhou QL, Shen W. [Regulatory mechanism of malignant behavior of endometriosis mediated by puerarin]. $J$ Chin Integr Med. 2009;7(1):43-47. Chinese.

9. Shoji T, Takatori E, Murakami K, et al. A case of ovarian adenosquamous carcinoma arising from endometrioid adenocarcinoma: a case report and systematic review. J Ovarian Res. 2016;9(1):48-54.

10. Xu L, Zhou QL, Han J, et al. Effects of Chinese herbal medicine Neiyi Recipe-medicated serum on angiopoiesis of endometriosis in the chick chorioallantoic membrane model. J Chin Integr Med. 2012;10(7): 800-806.

11. McClung EC, Wenham RM. Profile of bevacizumab in the treatment of platinum-resistant ovarian cancer: current perspectives. Int $J$ Womens Health. 2016;8:59-75.

12. Zhang M, Tian Z, Sun Y. Successful treatment of ovarian cancer with apatinib combined with chemotherapy. Medicine (United States). 2017; 96(45):45-49.

13. Deng L, Wang Y, Lu W, Liu Q, Wu J, Jin J. Apatinib treatment combined with chemotherapy for advanced epithelial ovarian cancer: a case report. Onco Targets Ther. 2017;10:1521-1525.

14. du Bois A, Floquet A, Kim JW, et al. Incorporation of pazopanib in maintenance therapy of ovarian cancer. J Clin Oncol. 2014;32(30): 3374-3382.

15. El Helali A, McCabe N, Dickson N, et al. Acquired platinum resistance enhances tumour angiogenesis through activation of vascular mimicry. Cancer Res. 2017;77(13):152-158.

16. Kim G, Ison G, McKee AE, et al. FDA approval summary: olaparib monotherapy in patients with deleterious germline BRCA-mutated advanced ovarian cancer treated with three or more lines of chemotherapy. Clin Cancer Res. 2015;21(19):4257-4261.

17. McLachlan J, Banerjee S. Olaparib for the treatment of epithelial ovarian cancer. Expert Opin Pharmacother. 2016;17(7):995-1003.

18. Hazarika M, Chuk MK, Theoret MR, et al. U.S. FDA approval summary: nivolumab for treatment of unresectable or metastatic melanoma following progression on ipilimumab. Clin Cancer Res. 2017;23(14): 3484-3488.

19. Barone A, Hazarika M, Theoret MR, et al. FDA approval summary: pembrolizumab for the treatment of patients with unresectable or metastatic melanoma. Clin Cancer Res. 2017;23(19):5661-5665.

20. Pai-Scherf L, Blumenthal GM, Li H, et al. FDA approval summary: pembrolizumab for treatment of metastatic non-small cell lung cancer: first-line therapy and beyond. Oncologist. 2017;22(11):1392-1399.

21. Varga A, Piha-Paul SA, Ott PA, et al. Pembrolizumab in patients (pts) with PD-L1-positive (PD-L1+) advanced ovarian cancer: updated analysis of KEYNOTE-028. J Clin Oncol. 2017;35(15):5513. 
22. Wenham RM, Dorman D, Lee JK, Apte SM, Shahzad MM, Chon HS Phase 2 trial of dose dense (weekly) paclitaxel with pembrolizumab in platinum resistant recurrent ovarian cancer. Int J Gynecol Cancer. 2016;26:877-881.

23. Matulonis UA, Chen M, Puhlmann M, Shentu Y, Ledermann J. KEYNOTE-100: phase 2 trial of pembrolizumab in patients with advanced recurrent ovarian cancer. Ann Oncol. 2016;27(Suppl 6):296-312.

24. Hamanishi J, Mandai M, Ikeda T, et al. Safety and antitumor activity of anti-PD-1 antibody, nivolumab, in patients with platinum-resistant ovarian cancer. J Clin Oncol. 2015;33(34):4015-4022.

25. Sun LM, Liu YC, Li W, et al. Nivolumab effectively inhibit platinumresistant ovarian cancer cells via induction of cell apoptosis and inhibition of ADAM17 expression. Eur Rev Med Pharmacol Sci. 2017;21(6): 1198-1205.

26. Prasad V, Kaestner V. Nivolumab and pembrolizumab: monoclonal antibodies against programmed cell death-1 (PD-1) that are interchangeable. Semin Oncol. 2017;44(2):132-135.
27. Zhu X, Lang J. Programmed death-1 pathway blockade produces a synergistic antitumor effect: combined application in ovarian cancer. J Gynecol Oncol. 2017;28(5):e64.

28. Guan X, Wang H, Ma F, Qian H, Yi Z, Xu B. The efficacy and safety of programmed cell death 1 and programmed cell death 1 ligand inhibitors for advanced melanoma: a meta-analysis of clinical trials following the PRISMA guidelines. Medicine (United States). 2016;95(11):e3134.

29. Strickland KC, Howitt BE, Shukla SA, et al. Association and prognostic significance of BRCA1/2-mutation status with neoantigen load, number of tumor-infiltrating lymphocytes and expression of PD-1/PD-L1 in high grade serous ovarian cancer. Oncotarget. 2016;7(12):13587-13598.

30. Jiao S, Xia W, Yamaguchi H, et al. PARP inhibitor upregulates PD-L1 expression and enhances cancer-associated immunosuppression. Clin Cancer Res. 2017;23(14):3711-3720.

31. Lim JSJ, Tan DSP. Understanding resistance mechanisms and expanding the therapeutic utility of PARP inhibitors. Cancers. 2017; 9(8):109-123.

\section{Publish your work in this journal}

OncoTargets and Therapy is an international, peer-reviewed, open access journal focusing on the pathological basis of all cancers, potential targets for therapy and treatment protocols employed to improve the management of cancer patients. The journal also focuses on the impact of management programs and new therapeutic agents and protocols on

\section{Dovepress}

patient perspectives such as quality of life, adherence and satisfaction. The manuscript management system is completely online and includes a very quick and fair peer-review system, which is all easy to use. Visit http://www.dovepress.com/testimonials.php to read real quotes from published authors. 